\title{
Mechanism to Enhance Visible-light-driven Photocatalysis of Flower-like Composite of AgI Nanoparticle/BiOI Nanosheet
}

Jaafar Hasan ${ }^{\mathrm{a}, \mathrm{b}}$, Gaowei Ouyang ${ }^{\mathrm{a}}$, Jing Wang ${ }^{\mathrm{a}}$, Haidi Li ${ }^{\mathrm{a}}$, Guang Tian ${ }^{\mathrm{a}}$, Chuanguang Qin ${ }^{\mathrm{a} *}$

${ }^{a}$ Shaanxi Key Laboratory of Polymer Science and Technology, OME Key Laboratory of Supernomal Material Physics and Chemistry, Department of Chemistry, School of Chemistry and Chemical Engineering, Northwestern Polytechnical University, Xi'an, 710129, P. R. China.

${ }^{b}$ College of Dentistry, AL-Muthanna University, Samawah, AL-Muthanna, 00964, Province,Iraq

Corresponding author: qinchg@nwpu.edu.cn

\begin{abstract}
Nanosheet BiOI materials were prepared by using the precipitation-hydrothermal method. Moreover, nanoparticle AgI/BiOI nanosheet composites were prepared using an ion-exchange method and by controlling the amount of BiOI. The phase composition, optical morphology, and absorption properties of the samples were determined using XRD, SEM, XPS, TEM, HRTEM, and UV-Vis diffuse reflectance. Organic dyes, such as methyl orange (MO) and Coomassie brilliant blue R-250 (CBB), were used to check the photocatalytic performance of the composites when undergoing photodegradation in visible light. The prepared composites had high purity, and the AgI nanoparticles were evenly loaded on the flower-like BiOI nanosheets, and both can absorb the visible light. The photocatalytic activity of sole BiOI or AgI was poor, whereas that of the composites was much better. The composite with 50\% AgI exhibited the best photocatalytic activity because of the formation of a p-n heterojunction, which promotes the separation of photogenerated carriers and makes AgI stable under visible-light irradiation.
\end{abstract}

Keywords: BiOI; AgI; photocatalysis; visible-light-driven; heterojunction 


\section{Introduction}

Environmental pollution is becoming an increasingly serious problem, and measures to control pollution are needed. The solar photocatalytic oxidation method is a cleaner, simpler, and more economical and environmentally friendly approach for eliminating organic pollutants compared with traditional chemical oxidation ${ }^{1}$, physical adsorption ${ }^{2}$ and biodegradation ${ }^{3}$. Solar photocatalytic oxidation will help with the development of a future free of pollutants from factories and other sources. Conventional wide bandgap semiconductor photocatalytic materials, such as $\mathrm{TiO}_{2}$ and $\mathrm{ZnO}$, can only absorb ultraviolet light ${ }^{4-7}$, and the utilization rate of solar energy is very low. They also have high recombination rates of photoinduced electron-hole pairs. Thus, a photocatalyst that absorbs visible light needs to be developed.

Bismuth oxyhalides ( $\mathrm{BiOX}, \mathrm{X}=\mathrm{Cl}, \mathrm{Br}, \mathrm{I})$ are semiconductor photocatalysts with high activity under ultraviolet or visible light illumination. Bismuth oxy-iodine (BiOI) has the best photocatalytic activity among bismuth oxyhalides because of its suitable bandgap (about 1.7-1.9 eV) ${ }^{8}$. BiOI is a new visible-light catalyst with a low bandgap (1.7-1.9 eV). So, it absorbs a wide range of visible light 9,10 and has a special layered crystal structure. This feature means that photogenerated electron-hole pairs can be separated ${ }^{11}$. Thus, in recent years, researchers focused on BiOI. Although the photocatalytic activity of $\mathrm{BiOI}$ is relatively good, its limiting bandwidth is too small. The photogenerated electrons and recombination of electron holes are serious issues, as they lead to the reduced utilization of photogenerated carriers. Also, the low valence potential results in an insufficient capacity for oxidation, thereby reducing its photocatalytic activity ${ }^{12-14}$. $\mathrm{BiOI}$ needs to be modified in some way to prevent the combination of photogenerated electronholes pairs. Combining it with other semiconductor compounds is an effective method.

Supported-AgX $(\mathrm{X}=\mathrm{Cl}, \mathrm{Br}, \mathrm{I})$ has a strong visible light response, and surface plasmon resonance is present with the Ag nanoparticles ${ }^{15}$. Thus, researchers are showing an increasing amount of interest on photocatalyst silver halide composites. Compared with $\mathrm{AgCl}$ and $\mathrm{AgBr}, \mathrm{AgI}$ is rarely studied because of the sensitivity of AgI to light and its compound crystal structure. AgI is mostly used as a co-catalyst with other semiconductor materials to increase absorption in the visible light range. Based on theoretical calculations, the bandgaps of $\mathrm{AgCl}, \mathrm{AgBr}$, and $\mathrm{AgI}$ become increasingly narrow, and the absorption capacity and utilization rate of photons increase. At $420 \mathrm{~K}, \mathrm{AgI}$ is usually present in the stable $\beta$-phase (hexagonal quartzite structure) and metastable $\gamma$-phase (cubic sphalerite structure). At above $420 \mathrm{~K}$, the cubic $\alpha$-phase exists stably, 
according to relevant reports, and the $\beta$-phase of $\mathrm{AgI}$ has a higher photocatalytic activity than the $\alpha$-phase ${ }^{16}$.

Experiments proved that when AgI is supported by another semiconductor with photocatalytic activity, the efficiency of the photocatalytic activity of the semiconductor is effectively improved 17, 18. AgI has a narrow bandgap and strong absorption in visible light. The heterojunction structure of AgI-based composite semiconductors can effectively separate photogenerated electrons and holes, sensitize the semiconductor, and improve the photocatalytic efficiency ${ }^{19}$. Therefore, AgI and $\mathrm{BiOI}$ are combined to produce composite photocatalysts that show excellent performance levels. The $\mathrm{Ag}^{+}$reacts with $\mathrm{BiOI}$ to form $\mathrm{AgI}$, because the solubility product constant $\mathrm{Ksp}$ of $\mathrm{AgI}$ is smaller than that of $\mathrm{BiOI}^{20}$.

In this work, AgI nanoparticle (NP)/BiOI nanosheet (NS) composite photocatalysts were fabricated with various ratios of $\mathrm{AgI} \mathrm{NP} / \mathrm{BiOI} \mathrm{NS}$ via the hydrothermal method of precipitation and the ion-exchange method. The photocatalytic activity of the p-n heterojunction AgI/BiOI was assessed under visible light irradiation using the photocatalytic degradation of the organic contaminants, methyl orange, and Coomassie brilliant blue. Under visible light irradiation, $\mathrm{AgI} / \mathrm{BiOI}$ served as a very effective composite photocatalyst for the removal of organic pollutants. The activity of photocatalysis was determined by the quantity of AgI added to the photocatalyst, and the most desirable ratio was obtained at $50 \%$ of $\mathrm{AgI} / \mathrm{BiOI}$. The stability of the $50 \% \mathrm{AgI} / \mathrm{BiOI}$ photocatalyst was evaluated by recycling tests. Modifying the wide bandgap semiconductor AgI with p-type narrow bandgap semiconductors by ion exchange could become a popular strategy.

\section{Experimental procedure}

\subsection{Materials}

Bismuth nitrate pentahydrate $\mathrm{Bi}\left(\mathrm{NO}_{3}\right)_{3} \cdot 5 \mathrm{H}_{2} \mathrm{O}$, ethylene glycol $\left(\mathrm{C}_{2} \mathrm{H}_{6} \mathrm{O}_{2}\right)$, and silver nitrate $\left(\mathrm{AgNO}_{3}\right)$ were purchased from Guangdong Guanghua Sci-Tech Co., Ltd., China. Polyvinylpyrrolidone (PVP K30) was purchased from Shanghai Blue season Biotechnology Co., Ltd., China. Methyl orange (MO) and Coomassie brilliant blue R-250 (CBB) were purchased from Tianjin Kemiou Chemical Reagent Co., Ltd., in China. All the chemicals were of high analytical grade and used as received. 


\subsection{Preparation of BiOI nanosheet}

Firstly, $4 \mathrm{mmol}$ of $\mathrm{Bi}\left(\mathrm{NO}_{3}\right)_{3} \cdot 5 \mathrm{H}_{2} \mathrm{O}$ was added to $40 \mathrm{~mL}$ of absolute ethanol to form a white microemulsion, which was then stirred at room temperature for $30 \mathrm{~min}$ and labeled as solution A. Then, $4 \mathrm{mmol}$ of KI was dissolved in $40 \mathrm{~mL}$ of distilled water to form solution B. Solution B was added dropwise to solution A with vigorous stirring, and the resulting solution was stirred for a further $2 \mathrm{~h}$. This mixture was transferred into a $100 \mathrm{~mL}$ Teflon-lined high-temperature reactor and placed in a furnace at $180{ }^{\circ} \mathrm{C}$ for $2 \mathrm{~h}$. After the reaction, the solution was naturally cooled to ambient temperature, the supernatant were discarded, and the remaining precipitate was washed and centrifuged with water and ethanol several times. The final product was dried at $60{ }^{\circ} \mathrm{C}$ for 12 $\mathrm{h}$ in a vacuum oven to produce the desired product.

\subsection{Preparation AgI nanoparticle/BiOI nanosheet composite}

Firstly, PVP K30 at $0.2 \mathrm{~g}$ was added to $100 \mathrm{~mL}$ of ethylene glycol. After sufficient dissolution, a certain mass of solid $\mathrm{AgNO}_{3}$ at $0.1,0.3,0.5$, or $0.7 \mathrm{mmol}$ was added. Stirring was conducted to sufficiently dissolve the $\mathrm{AgNO}_{3}$. Then, $1 \mathrm{mmol}$ of the $\mathrm{BiOI}$ sample was added to the abovementioned solution, which was sonicated for $15 \mathrm{~min}$ and further stirred vigorously for $2 \mathrm{~h}$. The resulting product was collected by centrifugation, washed with anhydrous ethanol three times. The obtained product was further dried overnight at $60{ }^{\circ} \mathrm{C}$ to obtain $\mathrm{AgI} / \mathrm{BiOI}$ compounds. AgI accounted for $10 \%, 30 \%, 50 \%$, and $70 \%$ of the total mass of the obtained $\mathrm{AgI} / \mathrm{BiOI}$ compounds.

\subsection{Characterization of structure and morphology}

The prepared catalysts were characterized by X-ray powder diffraction (XRD, D2 PHASER X) using $\mathrm{Cu} \mathrm{k} \alpha$ as the irradiation source $(\lambda=1.54178 \AA)$ in the range of the wide-angle data from 20 to $80(2 \theta)$ at $5^{\circ} \mathrm{min}^{-1}$ scan speed. The morphologies of the catalysts were observed by scanning electron microscopy (SEM, TM4000PLUS). The electronic states and chemical components were analyzed by X-ray photoelectron spectra (XPS, Axis Supra). The TEM and RHTEM (FEI Talos F200X TEM) confirmed the structural details of the prepared catalysts. The light absorption properties of the samples were analyzed by using a UV-Vis spectrophotometer (UV1800, scanning range: 200-800 nm) from Jinghua Instruments Co., Ltd. (Shanghai, China). A photochemical 
reactor equipped with $1000 \mathrm{~W}$ adjustable Xe lamp as a visible light source was analyzed by DSGHX-V, Dusi Instruments Co., Ltd. (Shanghai, China).

\subsection{Photocatalytic activity of the composites on organic dyes}

In this paper, methyl orange (MO) and Coomassie brilliant blue R-250 (CBB) were selected as the target degradation substrates. First, $10 \mathrm{mg} / \mathrm{L}$ of $\mathrm{MO}$ or $20 \mathrm{mg} / \mathrm{L}$ of $\mathrm{CBB}$ and $20 \mathrm{mg}$ of the catalyst were dissolved in $50 \mathrm{~mL}$ of solution concentration. Then, this solution was placed in a colorimetric quartz tube and kept in the dark to achieve an adsorption-desorption balance between the catalyst and the dye. Afterward, the solution was stirred for $30 \mathrm{~min}$. Subsequently, the dye and the catalyst were reacted under a $500 \mathrm{~W}$ xenon lamp $(\lambda>420 \mathrm{~nm})$ while being magnetically stirred. A sample was collected every $10 \mathrm{~min} ; 4 \mathrm{~mL}$ of the solution was withdrawn and centrifuged at high speed to remove catalyst particles. Then, a UV-Vis spectrophotometer was used to measure the absorbance of the supernatant and calculate its degradation rate. For the recycling experiment, the used $\mathrm{AgI} / \mathrm{BiOI}$ photocatalyst was separated from the solution by centrifugation, dried for $12 \mathrm{~h}$ at $60{ }^{\circ} \mathrm{C}$, and reused to decompose $\mathrm{MO}$ and $\mathrm{CBB}$ at the same concentration. The efficiency of degradation (\%) was determined as follows:

$$
\text { Degradation }(\%)=\left[\mathrm{C}_{\mathrm{o}}-\mathrm{C}_{\mathrm{t}} / \mathrm{C}_{\mathrm{o}}\right] \times 100
$$

Where $C_{o}$ the initial concentration of organic dyes and $C_{t}$ is the concentration of organic dyes at time t.

\section{Results and discussion}

\subsection{Formation mechanism of the AgI nanoparticle/BiOl nanosheet composite}

The process and mechanism to form the AgI nanoparticle/BiOI nanosheet composite photocatalyst were shown as Fig. 1. First, uniform BiOI samples were prepared by the precipitation-hydrothermal technique. Many latticed 2D nanosheets composed the BiOI flowers. Then AgI nanoparticles were created and deposited during the ion-exchange reaction between $\mathrm{BiOI}$ and $\mathrm{Ag}^{+}$in the aqueous solution above the surface of the BiOI nanosheets. These nanoparticles were highly dispersed, and the generated $\mathrm{BiO}^{+}$ions were dissolved in the solvent.

$\mathbf{B i O I}+\mathbf{A g}^{+} \rightarrow \mathbf{A g I} \downarrow+\mathbf{B i O}^{+}$ 


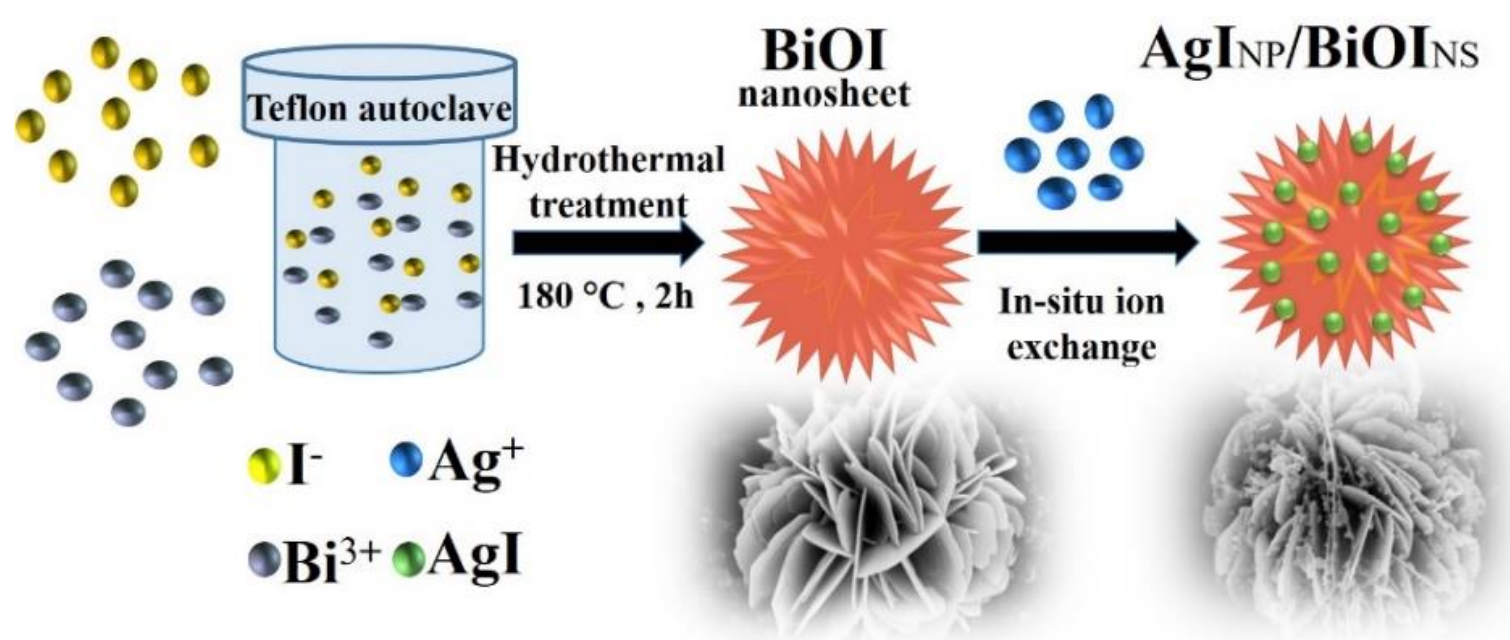

Fig. 1 Schematic description of the proposed AgI nanoparticle/BiOI nanosheet composite forming process.

\subsection{Crystal structure}

The XRD patterns of the AgI NP/ BiOI NS composites with different ratios were shown in Fig. 2. The diffraction peaks of all the samples were relatively sharp and strong, indicating the high crystallinity of the as-prepared products. No miscellaneous peaks were observed, indicating that the products were relatively pure. Compared with the standard spectrum, the XRD pattern of BiOI was consistent with the standard spectrum JCPDS No.10-0445, which was tetragonal. When $\mathrm{BiOI}$ was replaced by $\mathrm{Ag}^{+}$, the diffraction peaks of $\mathrm{AgI}$ appeared. When only $10 \%$ of $\mathrm{BiOI}$ was replaced, the diffraction peaks of $\mathrm{AgI}$ were not observed clearly, indicating that the content of $\mathrm{BiOI}$ incorporated into the compound was low. When the molar amount of BiOI was further increased to $30 \%$, the diffraction peaks of $\mathrm{AgI}$ was observed. When the molar amount of BiOI was increased to $50 \%$ and $70 \%$, the diffraction peaks of $\mathrm{AgI}$ were more pronounced. Compared with the standard spectrum, the XRD pattern of AgI was found to be consistent with the standard spectrum JCPDS No.09-0374, which was hexagonal, and the AgI was $\beta$-AgI. 


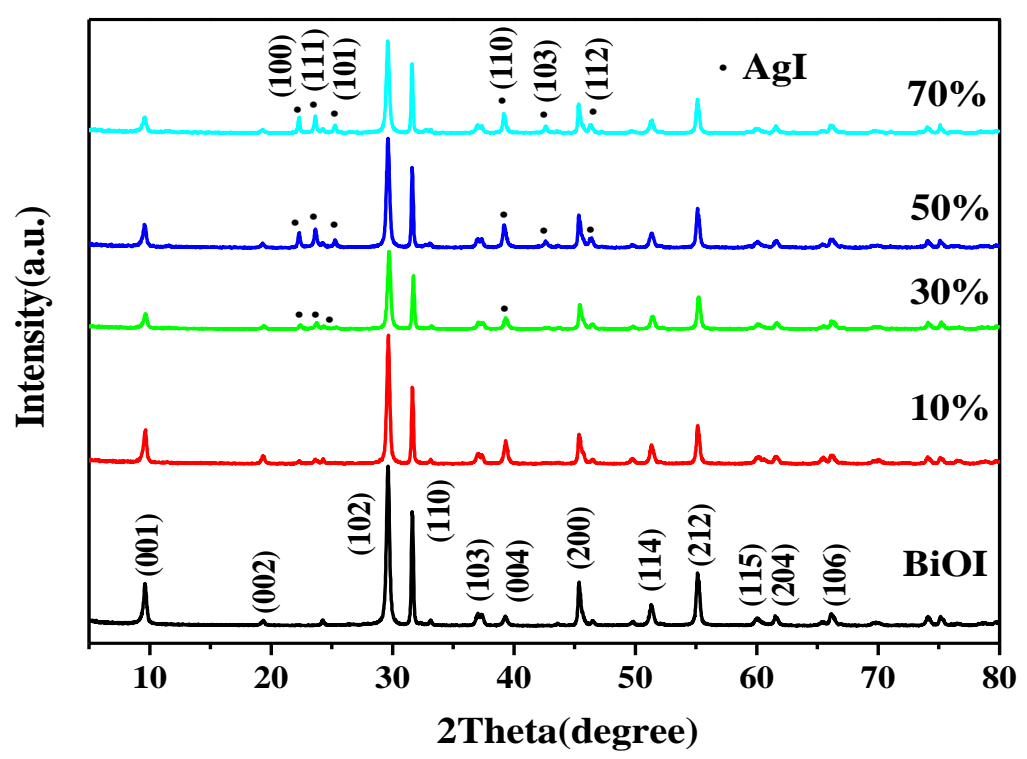

Fig. 2 Powder X-ray diffraction patterns of AgI NP / BiOI NS composites with different proportions.

\subsection{SEM analysis}

SEM was performed to investigate the morphological features of the samples. Fig. 3 shows the images of the AgI NP /BiOI NS composites with different ratios. Fig. 3a and 3b are SEM images of the pure $\mathrm{BiOI}$ samples at different magnifications. The figures show that the $\mathrm{BiOI}$ flowers are relatively uniform and assembled as sheets $4.5 \mu \mathrm{m}$ in length and $1 \mu \mathrm{m}$ wide. Fig. $3 \mathrm{c}$ and $3 \mathrm{~d}$ show different magnifications of the $10 \% \mathrm{AgI} / \mathrm{BiOI}$ compound SEM images. The morphology of the flower-like BiOI remains intact, and the nano-AgI particles are uniformly attached to the surface. With increasing AgI composite ratio, the amount of AgI particles attached to the flower's surface increases, as shown in Fig. 3 (e-j). The figures also show that the prepared AgI nanoparticles are flaky and extremely irregular in shape, and the sizes are between 60 and $100 \mathrm{~nm}$. Moreover, some of the AgI nanoparticles are agglomerated. 

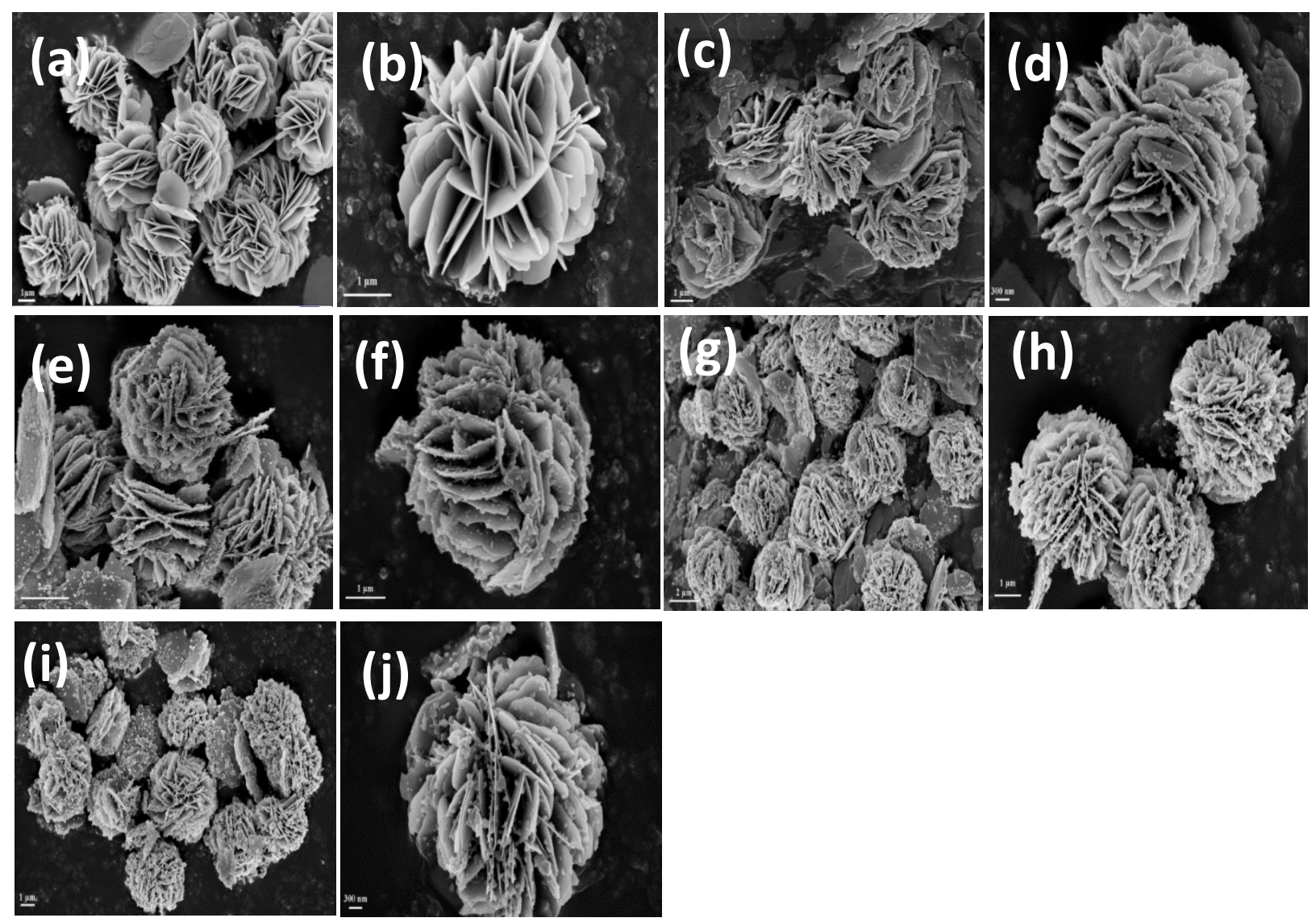

Fig. 3 SEM micrographs of AgI NP/BiOI NS composites of various proportions: (a and b) pure BiOl; (c and d) AgI NP/BiOI NS composites of 10\%; (e and f) AgI NP/BiOI NS composites of 30\%; (g and h) AgI NP/BiOI NS composites of 50\%; and ( $i$ and $j$ ) AgI NP/BiOI NS composites of $70 \%$.

\subsection{XPS analysis}

The chemical composition and oxidation state of the 50\% AgI NP/BiOI NS composites were measured to further analyze the surface. XPS analysis was carried out. The results are shown in Fig. 4. The full spectrum of XPS (Fig. 4a) shows that the elements contained in the sample are C, $\mathrm{O}, \mathrm{Bi}, \mathrm{I}$, and $\mathrm{Ag}$. The $\mathrm{C}$ element is due to the introduction of organic matter onto the conductive adhesive and not the sample itself. In addition, no other elements are present, indicating that the prepared AgI/BiOI compound sample has a high level of purity. Fig. 4(b-e) showed the survey spectrum of Bi 4f, O 1s, I 3d, and Ag 3d. In Fig. 4b, the two strong peaks at 159.0 and $164.3 \mathrm{eV}$ are related to $\mathrm{Bi} 4 \mathrm{f}_{7 / 2}$ and $\mathrm{Bi} 4 \mathrm{f}_{5 / 2}$, respectively, which are the distinctive peaks of $\mathrm{Bi}^{3+}$ in $\mathrm{BiOI}^{21}$. 
As shown in Fig. 4c, the peak at $530.63 \mathrm{eV}$ could be related to the O1s region, which is attributed to the $\mathrm{Bi}-\mathrm{O}$ bond in the $\mathrm{BiOI}$ layered structure of the $\left[\mathrm{Bi}_{2} \mathrm{O}_{2}\right]$ layer ${ }^{22}$. Fig. $4 \mathbf{d}$ shows the highresolution spectrum of $\mathrm{I} 3 \mathrm{~d}$, and the two peaks at 629.35 and $617.85 \mathrm{eV}$ belong to $\mathrm{I} 3 \mathrm{~d}_{3 / 2}$ and I $3 d_{5 / 2}$, respectively, which can be attributed to $\mathrm{I}^{-23}$. The binding energy values of $\mathrm{Ag} 3 \mathrm{~d}_{5 / 2}$ and $\mathrm{Ag}$ $3 \mathrm{~d}_{3 / 2}$ were noted at 366.9 and $372.9 \mathrm{eV}$, respectively, in Fig. 4e, suggesting that $\mathrm{Ag}^{+} \mathrm{or} \mathrm{Ag}^{0}$ should form as $\mathrm{Ag}$ in the compound ${ }^{24}$. Nevertheless, the XPS results confirmed that AgI and BiOI coexist in the $\mathrm{AgI} / \mathrm{BiOI}$ compounds and that $\mathrm{Ag}$ or I is not correlated with other impurities. This finding is consistent with the findings from the XRD analysis.
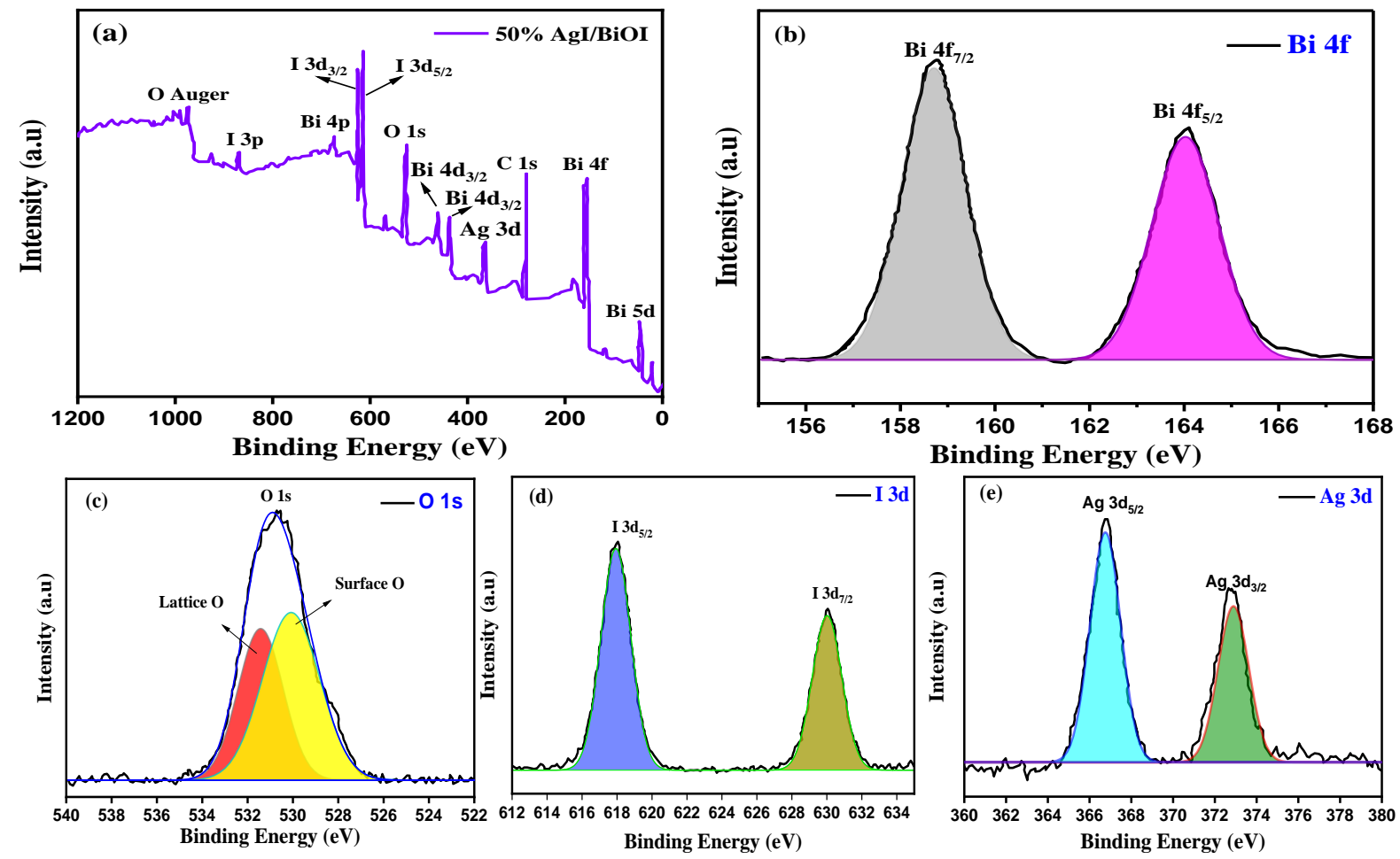

Fig. 4 XPS spectra of $50 \%$ AgI NP/BiOI NS composite survey spectrum (a), high-resolution XPS spectra of Bi $4 f(b), O$ 1s (c), I 3d (d), and Ag 3d (e).

\subsection{UV-VIS DRS analysis}

To analyze the light absorption properties of the samples, the UV-visible diffuse reflectance spectra of the three samples of $\mathrm{BiOI}, \mathrm{AgI}$, and the 50\% $\mathrm{AgI} / \mathrm{BiOI}$ compounds are shown in Fig. 5a. The three samples are absorbed in the visible light region. The absorption edges of BiOI and 
AgI are 653 and $454 \mathrm{~nm}$, respectively. The absorption edge of the 50\% AgI/BiOI compound is close to BiOI and slightly shifted red, showing that the light absorption range of the sample expands after the compound, and the visible light utilization increases. In addition, for the semiconductor materials, the forbidden bandwidth energy gap $(\mathrm{Eg})$ can be obtained from equation (2) as follows:

$$
\alpha h v=c(h v-E g)^{\mathrm{n} / 2}
$$

where $\alpha$ is the absorption coefficient, $\mathrm{h}$ is the Planck constant $\left(\mathrm{J}^{\mathrm{s}-1}\right), v$ is the incident light frequency $(\mathrm{Hz}), c$ is the proportional coefficient, and Eg is the forbidden bandgap energy. $\mathrm{n}$ depends on the nature of the semiconductor, where the direct bandgap semiconductor is 1 , and the indirect bandgap semiconductor $\mathrm{n}$ is $4^{25}$. According to the literature, BiOI is an indirect bandgap semiconductor that takes the $\mathrm{n}$ value of $4^{26}$. AgI is a direct bandgap semiconductor that takes the $\mathrm{n}$ value of $1^{16}$. Therefore, equation (2) can be transformed into equations (3) and (4):

$$
\begin{aligned}
& (\alpha h v)^{1 / 2}=C(h v-E g) \\
& (\alpha h v)^{2}=C(h v-E g)
\end{aligned}
$$
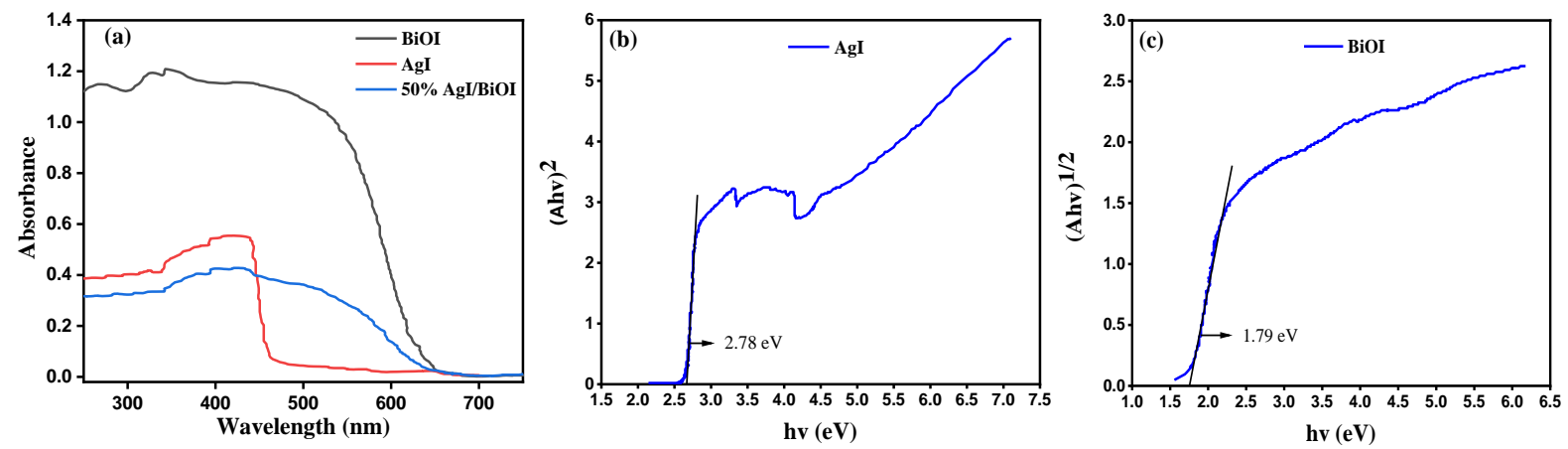

Fig. 5 Distribution of UV reflectance spectra from three different samples (a). Tauc plot of $(\alpha h v)^{2}$ versus energy (hv) of Agl (b) and (ahv) ${ }^{1 / 2}$ versus energy (hv) of BiOl (c) for the bandgap energy.

The light absorption coefficient $\alpha$ and the absorbance $\mathrm{A}$ value are proportional to the use of $\mathrm{A}$ instead of $\alpha$. BiOI is plotted with $(\mathrm{Ah} v)^{1 / 2}$ for hv, whereas $\mathrm{AgI}$ is plotted with $(\mathrm{Ah} v)^{2}$ for hv, as shown in Fig. 5b and 5c. The horizontal axis intercept is the Eg. The bandgaps (Eg) of the BiOI and $\mathrm{AgI}$ samples are 1.79 and $2.78 \mathrm{eV}$, respectively. 


\subsection{TEM analysis}

To elucidate the structure of the sample and the interplay between $\mathrm{AgI}$ and $\mathrm{BiOI}$, the $50 \% \mathrm{AgI}$ NP/BiOI NS was examined using transmission electron microscopy (TEM) and high-resolution transmission electron microscopy (HRTEM). Fig. 6a shows a clear TEM image of AgI/BiOI, in which the dispersal of the AgI molecules on the BiOI surface is irregular. Fig. $6 \mathbf{b}$ presents a highresolution image showing lattice fringes of $0.312 \mathrm{~nm}$, which is in accordance with the (102) crystal plane of BiOI. The lattice fringes with a spacing of $0.26 \mathrm{~nm}$ are compatible with the (101) crystal
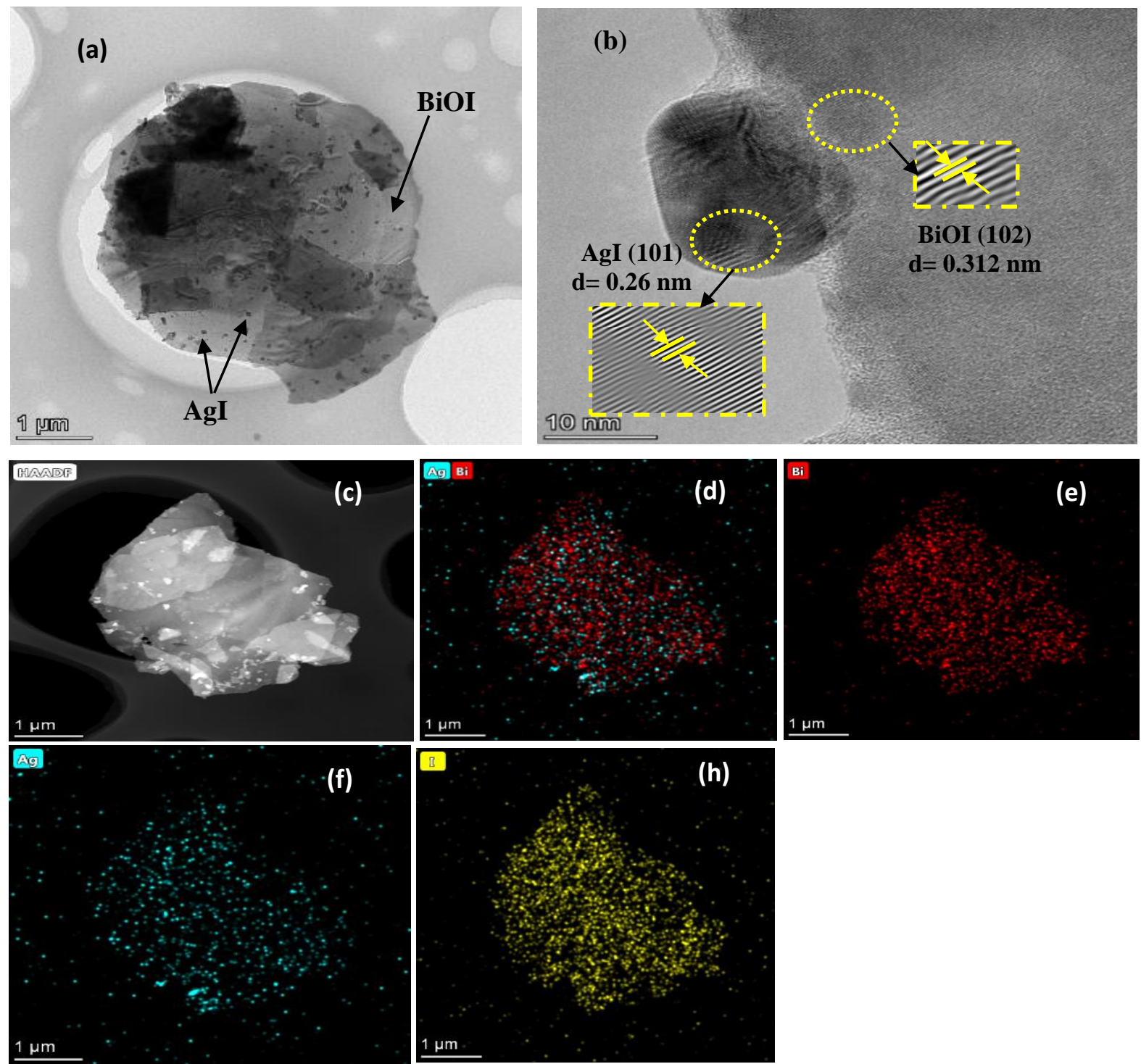

Fig. 6 An image of 50\% Agl/BiOl composite from (a) TEM and (b) HRTEM. The elemental mapping images of $50 \% \mathrm{Agl} / \mathrm{BiOl}$ composite with a scale bar of $1 \mu \mathrm{m}(\mathrm{c}) ; \mathrm{Ag}$ and $\mathrm{Bi}(\mathrm{d}), \mathrm{Bi}(\mathrm{e}), \mathrm{Ag}(\mathrm{f})$, and I (h). 
plane of AgI. These results suggest that the interplay between AgI and BiOI, namely, AgI NP/BiOI NS, consists of lattice fringes between the (102) plane of BiOI and the (101) plane of AgI, which corresponds with the obtained XRD patterns in Fig. 2. The element mapping images from the TEM testing of the AgI NP/BiOI NS composites are shown in Fig. 6 (c-h). Moreover, the distributions of the elements $\mathrm{Bi}, \mathrm{Ag}$, and $\mathrm{I}$ in the $\mathrm{AgI} / \mathrm{BiOI}$ composite are shown. Element mapping reveals that the $\mathrm{Bi}$ and I elements are equally dispersed in the compound, indicating that the AgI and BiOI are well distributed in the composites.

\subsection{Photocatalytic performance and activity analysis}

To further investigate the photocatalytic performance of the AgI nanoparticle/BiOI nanosheet composites with different ratios, photocatalytic degradation experiments were carried out to compare the efficiency of the degradation of MO and CBB. Fig. 7 (a1 and a2) shows that in the visible light irradiation, $\mathrm{MO}$ and $\mathrm{CBB}$ at low amounts are decomposed. The photocatalytic activity of the pure flower BiOI was low, and about $12.7 \%$ and $13 \%$ of $\mathrm{MO}$ and CBB were degraded in 30 min, respectively. However, the pure AgI photocatalytic activity was lower. Only $6.2 \%$ and $6.6 \%$ of the $\mathrm{MO}$ and $\mathrm{CBB}$ were degraded in $30 \mathrm{~min}$, respectively. This result was due to the instability of $\mathrm{AgI}$ in light and the easy production of elemental silver. However, after integrating the $\mathrm{AgI} / \mathrm{BiOI}$ compounds, the photocatalytic efficiency was greatly improved. Based on the molar ratios of 10 , 30,50 , and $70 \%$ of the $\mathrm{AgI} / \mathrm{BiOI}$ composites, the proportions of $\mathrm{MO}$ degraded in 30 min were $59.0 \%, 90.7 \%, 96.9 \%$, and $81.4 \%$, respectively, whereas the proportions of CBB degraded in 30 $\min$ were $42.0 \%, 47.3 \%, 97 \%$, and $75.5 \%$, respectively. The degradation activities of MO and $\mathrm{CBB}$ increased first and then decreased with increasing AgI ratio. They reached the maximum levels when the ratio of AgI was 50\%, as shown in Fig. 7 (b1 and b2).

Obviously, the photocatalytic process of the organic pollutants $\mathrm{MO}$ and $\mathrm{CBB}$ adheres to the apparent pseudo-first-order kinetics ${ }^{27}$. The kinetic photocatalytic degradation of all the two dyes was studied by using the follow equation (5):

$$
\operatorname{Ln}\left(\frac{\mathrm{C}}{\mathrm{C}_{\mathrm{o}}}\right)=\mathrm{K}_{\mathrm{app}} t
$$

where the $\mathrm{K}_{\text {app }}$ is the pseudo-first-order rate constant, $\mathrm{C}_{\mathrm{o}}$ is the initial concentration of dye, and $\mathrm{C}$ is the concentration of dye after photocatalytic degradation during time t. The degradation constant of $50 \% \mathrm{AgI} / \mathrm{BiOI}$ was $0.127 \mathrm{~min}^{-1}$, which was approximately 4 and 7.5 times higher than those of 
BiOI $\left(0.0322 \mathrm{~min}^{-1}\right)$ and $\mathrm{AgI}\left(0.017 \mathrm{~min}^{-1}\right)$, respectively, as seen in Fig. $\mathbf{7 c 1}$ for the MO dye. The degradation constant of the $50 \% \mathrm{AgI} / \mathrm{BiOI}$ composite $\left(0.130 \mathrm{~min}^{-1}\right)$ was found to be approximately 11 and 13 times higher than those of BiOI $\left(0.011 \mathrm{~min}^{-1}\right)$ and $\mathrm{AgI}\left(0.01 \mathrm{~min}^{-1}\right)$, respectively, as shown in Fig. 7c2 for the CBB dye. The highest levels of photocatalytic activity were observed after $30 \mathrm{~min}$ of irradiation. The prepared 50\% AgI NP/BiOI NS composites had excellent photocatalytic activity within the visible light region.
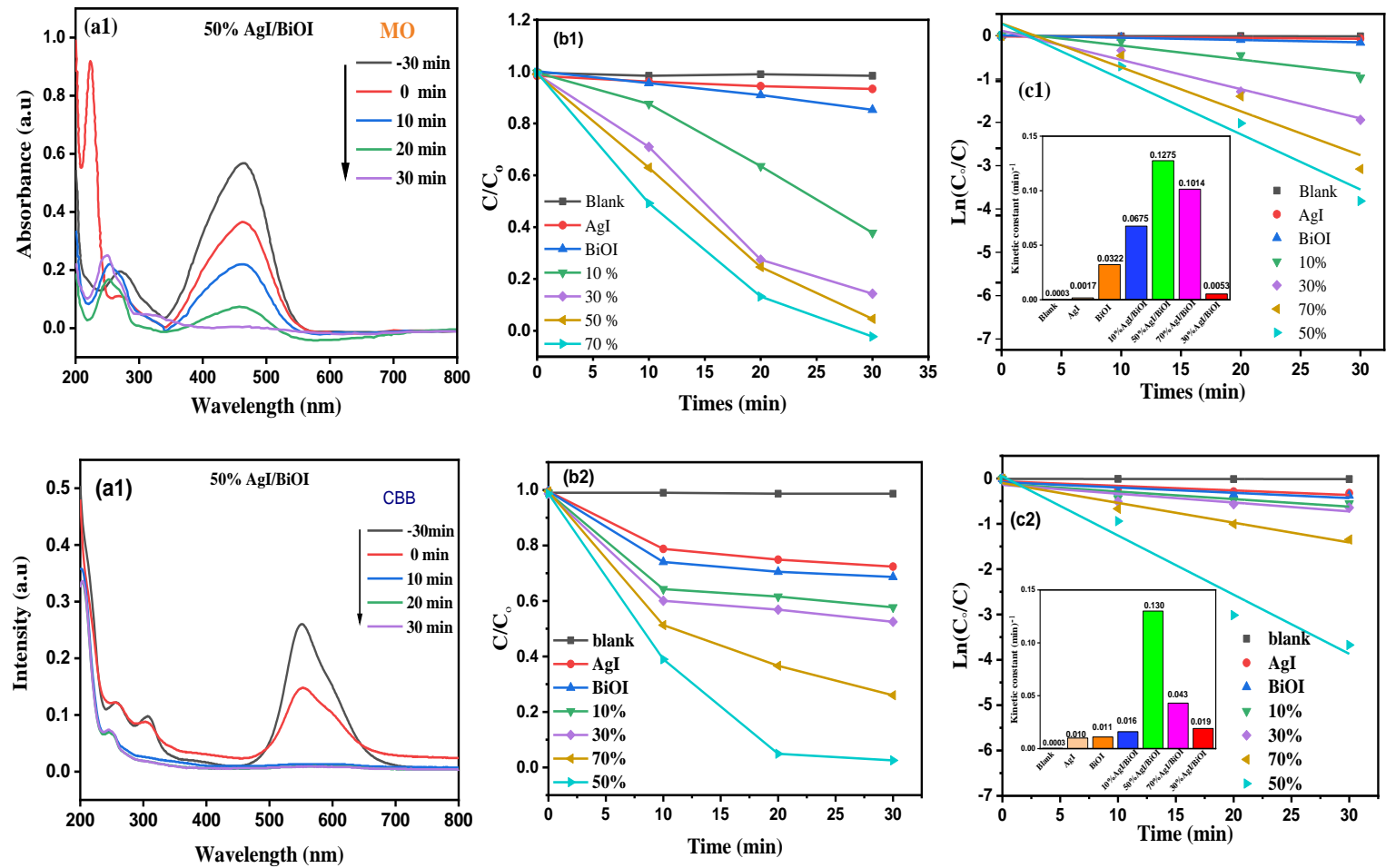

Fig. 7 ( $\mathrm{a} 1$ and $\mathrm{a} 2$ ) the UV-Vis absorption spectra of 50\% Agl/BiOl composite for MO and CBB dye; (b1 and b2) the degradation efficiency of $\mathrm{MO}$ and $\mathrm{CBB}$ dye in different proportions of $\mathrm{AgI} / \mathrm{BiOl}$ composite; and (c1 and $c 2$ ) the degradation curves of kinetics constant $\operatorname{Ln}\left(\mathrm{C}_{\mathrm{o}} / \mathrm{C}\right)$ of $\mathrm{MO}$ and $\mathrm{CBB}$ dye for different times.

We summarized the photocatalysts reported by other scientists in the literature in Table $\mathbf{1}$. The 50\% AgI NP/BiOI NS composite showed optimum performance for organic dye decomposition under visible light irradiation when compared with similar photocatalysts due to the novel structure and excellent separation performance of photo-generated electrons and holes in the 50\% AgI 
NP/BiOI NS composite. The composite achieved the highest rate of degradation efficiency in the shortest possible time when compared with the findings of previous studies.

Table 1. The degradation efficiency of different materials towards dye degradation under visiblelight irradiation (Xe lamp).

\begin{tabular}{|c|c|c|c|c|c|c|}
\hline No & Material & $\begin{array}{l}\text { Dosage of } \\
\text { catalyst }(\mathrm{mg} / \mathrm{L})\end{array}$ & $\begin{array}{l}\text { Organic } \\
\text { dye }\end{array}$ & $\begin{array}{l}\text { Irradiation } \\
\text { time (min) }\end{array}$ & $\begin{array}{l}\text { Rate of } \\
\text { degradation } \%\end{array}$ & Ref. \\
\hline \multirow[t]{2}{*}{1} & \multirow{2}{*}{$\begin{array}{l}\text { AgI NP/BiOI NS } \\
\text { Composite }\end{array}$} & 30 & $\mathrm{MO}$ & 30 & 96.9 & This work \\
\hline & & 30 & $\mathrm{CBB}$ & 30 & 97.0 & This work \\
\hline 2 & $\mathrm{BiOI} / \mathrm{BiPO}_{4}$ & 100 & MO & 120 & 98.0 & 28 \\
\hline 3 & $\begin{array}{l}\mathrm{MoS}_{2} / \mathrm{BiOI} / \mathrm{AgI} \\
\text { nanocomposit }\end{array}$ & 100 & $\mathrm{RhB}$ & 50 & 96.0 & 29 \\
\hline 4 & $\begin{array}{l}\mathrm{AgI} / \mathrm{BiOI} \\
\text { composite }\end{array}$ & 50 & $\mathrm{MB}$ & 20 & 95.3 & 21 \\
\hline 5 & $\begin{array}{l}\mathrm{BiOI} / \mathrm{BiOBr} \\
\text { heterostructures }\end{array}$ & 100 & MO & 600 & 90.0 & 10 \\
\hline 6 & $\begin{array}{l}\text { BiOI } \\
\text { nanostructures }\end{array}$ & 30 & $\mathrm{RhB}$ & 120 & 88.0 & 7 \\
\hline 7 & $\begin{array}{l}\mathrm{BiOI} / \mathrm{AgI} \\
\text { heterostructures }\end{array}$ & 100 & $\mathrm{RhB}$ & 90 & 83.0 & 30 \\
\hline 8 & $\begin{array}{l}\mathrm{AgI} / \mathrm{CuBi}_{2} \mathrm{O} \\
\text { heterojunction }\end{array}$ & 50 & $\mathrm{TC}$ & 80 & 80.0 & 31 \\
\hline 9 & AgI/BiOI hybrids & 2000 & PVP & 90 & 80.3 & 20 \\
\hline \multirow[t]{2}{*}{10} & \multirow{2}{*}{$\begin{array}{l}\mathrm{CQD} / \mathrm{BiOI} \\
\text { microspheres }\end{array}$} & 10 & RhB & 150 & 70.0 & \multirow[t]{2}{*}{22} \\
\hline & & 20 & $\mathrm{TC}$ & 120 & 50.0 & \\
\hline 11 & $\begin{array}{l}\mathrm{AgI} / \mathrm{BiOI} \\
\text { heterostructures }\end{array}$ & 1000 & MO & 150 & 75.0 & 18 \\
\hline 12 & $\begin{array}{l}\mathrm{BiOI} / \mathrm{BiOBr} \\
\text { heterojunction }\end{array}$ & 10 & MO & 300 & 63.10 & 32 \\
\hline 13 & $\begin{array}{l}\mathrm{BiOI} / \mathrm{BiOBr} \\
\text { heterostructures }\end{array}$ & 10 & MO & 300 & 61.20 & 33 \\
\hline
\end{tabular}




\subsection{Photoluminescence spectra}

The separation efficiency of electron-hole pairs plays a significant role in the capacity of the photocatalytic reaction. Photoluminescence spectra (PL) analysis is a technique used to inspect the separation efficiency of photo-generated charge carriers. A low PL intensity represents a decline in the recombination rate of the electron-hole pairs ${ }^{34}$. Fig. 8 shows the PL spectra of AgI, BiOI, and $50 \% \mathrm{AgI} \mathrm{NP} / \mathrm{BiOI} \mathrm{NS}$. The pure $\mathrm{AgI}$ and $\mathrm{BiOI}$ exhibit a normal emission with two peaks located at 480 and $580 \mathrm{~nm}$, respectively, whereas the PL spectra of the 50\% AgI NP/BiOI NS have other peaks at similar positions for the $\mathrm{AgI}$ and BIOI peaks but with lower intensity. The recombination of the electrons and holes is inhibited in the $50 \% \mathrm{AgI} \mathrm{NP} / \mathrm{BiOI}$ NS composite. Thus, it shows the best photocatalytic activity. If many electrons and holes are involved in the photocatalytic reaction, then the capacity of the photocatalytic activity is enhanced.

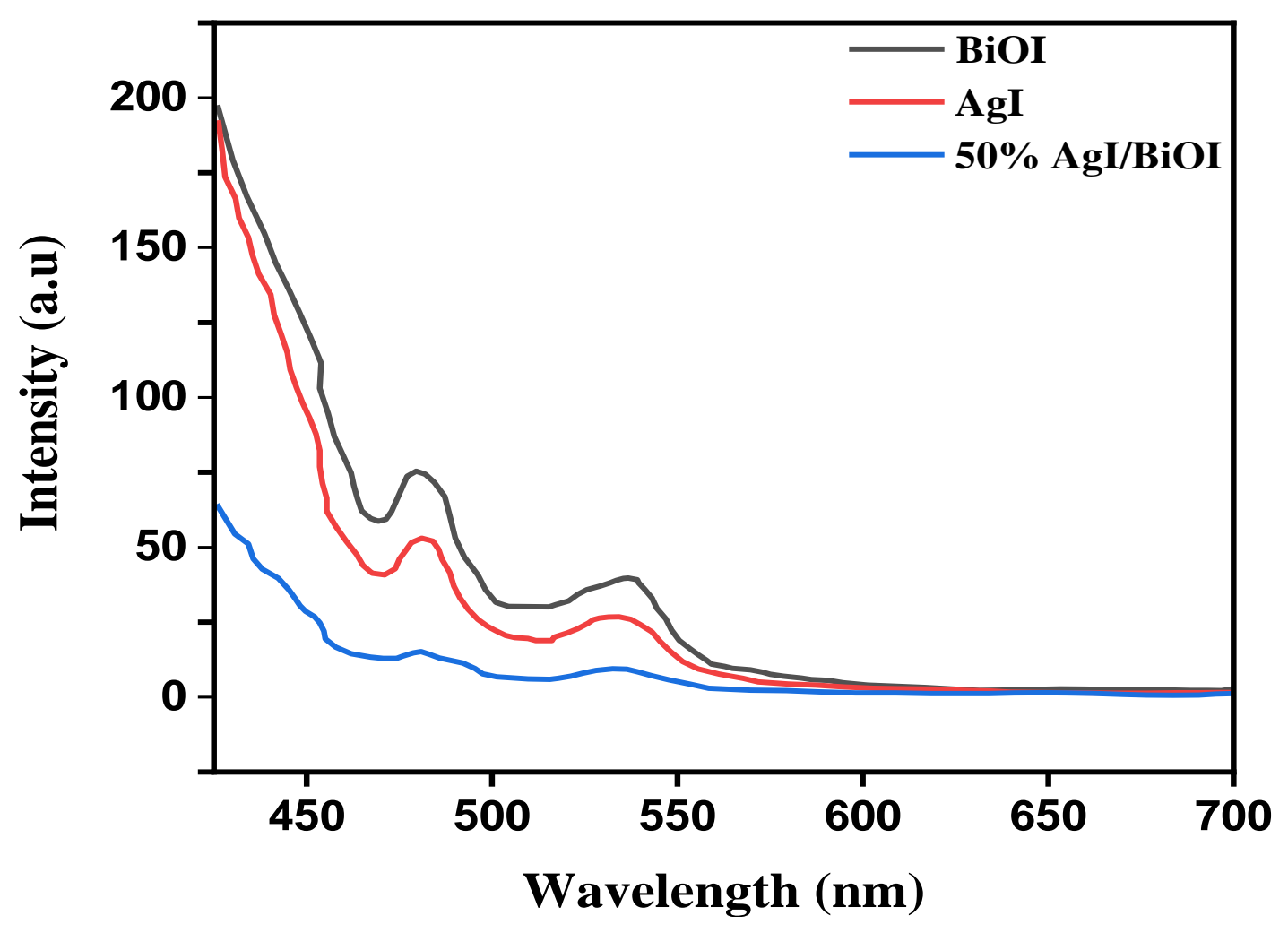

Fig. 8 PL spectrum of $\mathrm{BiOl}, \mathrm{Agl}$ and $50 \% \mathrm{AgI}$ NP/BiOI NS composite. 


\subsection{Stability of the AgI NP/BiOI NS composite catalyst}

$\mathrm{MO}$ and $\mathrm{CBB}$ degradation repeatability experiments were performed for the $50 \% \mathrm{AgI} / \mathrm{BiOI}$ to assess the stability of the $\mathrm{AgI} / \mathrm{BiOI}$ compounds. The $\mathrm{MO}$ and $\mathrm{CBB}$ degradation rates with the 50\% $\mathrm{AgI} / \mathrm{BiOI}$ were $95 \%$ and $95.5 \%$ after five recycling runs, respectively, as displayed in Fig. 9. High photocatalytic performance of $\mathrm{AgI} / \mathrm{BiOI}$ was maintained, thereby indicating that $\mathrm{AgI} / \mathrm{BiOI}$ was highly stable under visible light irradiation. These results indicated that $\mathrm{AgI} / \mathrm{BiOI}$ is somewhat stable when the organic dyes are photodegraded.

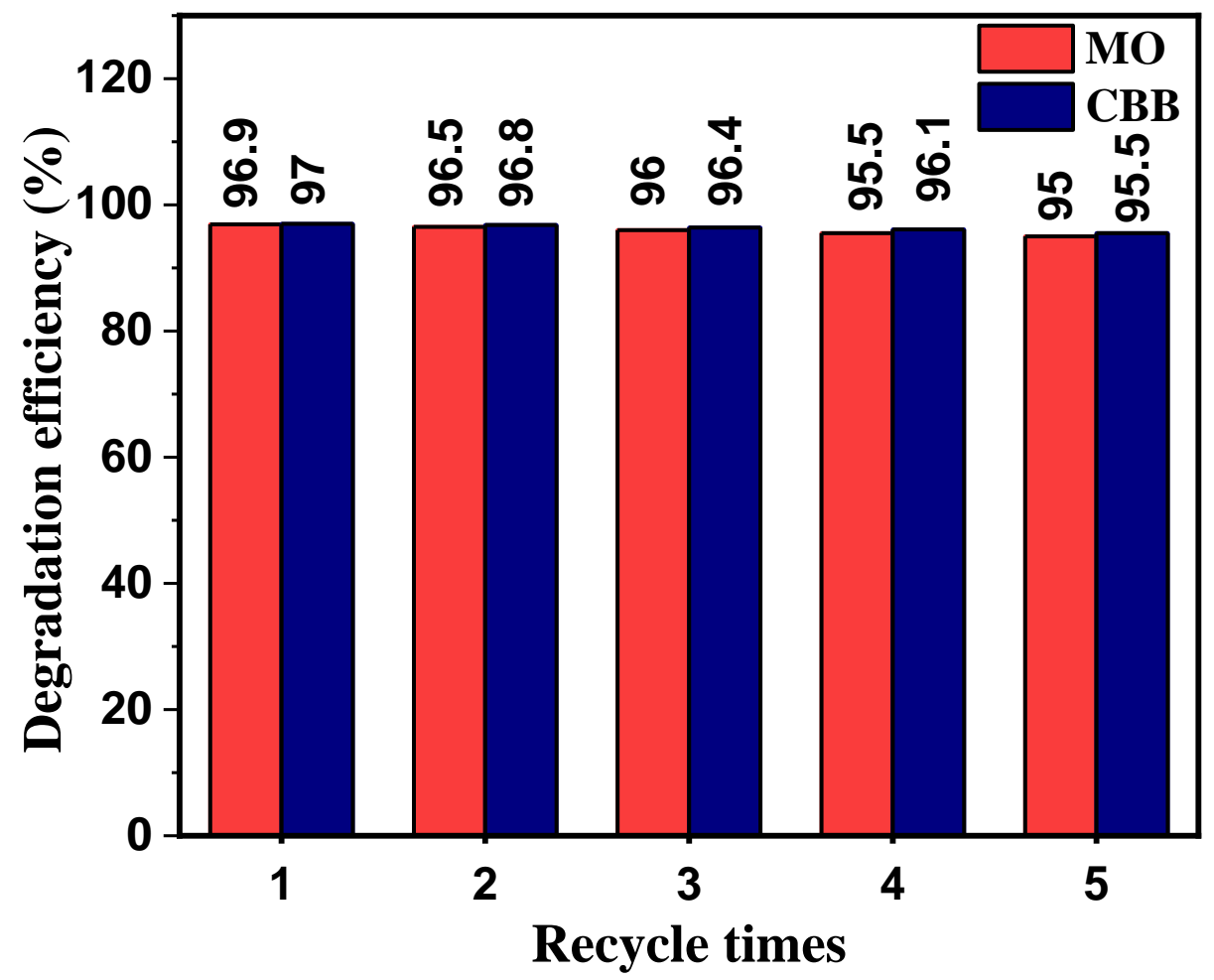

Fig. 9 Cycling runs for $\mathrm{MO}$ and CBB degradation at attending of 50\% AgI NP/BiOI NS Catalyst.

\subsection{The degradation mechanism of the AgI NP/BiOI NS composite catalyst}

The possible mechanism by which the organic pollutants are photodegraded on the AgI NP/BiOI NS composite photocatalysts is shown in Fig. 10. The valence band potential and the conduction band potential of the semiconductor $\mathrm{BiOI}$ and $\mathrm{AgI}$ can be calculated using the following equations ${ }^{35}:$ 


$$
\begin{aligned}
& E_{V B}=X-E^{e}+0.5 E_{g} \\
& E_{C B}=E_{V B}-E_{g}
\end{aligned}
$$

where $\mathrm{E}_{\mathrm{VB}}$ is the semiconductor valence band top potential, $\mathrm{X}$ is the electronegativity value of the semiconductor, $\mathrm{E}_{\mathrm{g}}$ is the forbidden bandwidth, $\mathrm{E}_{\mathrm{CB}}$ is the conduction band top potential, and $\mathrm{E}^{\mathrm{e}}$ is the surface electron free energy of the hydrogen atom (about $4.5 \mathrm{eV}$ ). $\mathrm{X}$ is obtained by taking the weighted average of the absolute electronegativity of the constituent semiconductor atoms, and the $\mathrm{X}$ values of $\mathrm{BiOI}$ and $\mathrm{AgI}$ are calculated to be 6.21 and $5.48 \mathrm{eV}$, respectively. According to equation (6), the $\mathrm{E}_{\mathrm{VB}}$ values of $\mathrm{BiOI}$ and $\mathrm{AgI}$ are 2.61 and 2.37eV, and based on equation (7), the $\mathrm{E}_{\mathrm{CB}}$ values are 0.82 and $-0.37 \mathrm{eV}$, respectively.

When the BiOI and AgI are coupled, both of them form a p-n heterojunction having the same Fermi level (EF) between the two sides, as shown in Fig. 10, because BiOI is a p-type semiconductor, and $\mathrm{AgI}$ is an n-type semiconductor. The $\mathrm{E}_{\mathrm{CB}}$ of $\mathrm{AgI}$ is lower than that of $\mathrm{BiOI}$. Thus, the electrons on the conduction band edge of the AgI easily flow through the interface into the conduction band edge of $\mathrm{BiOI}$, thereby preventing the $\mathrm{Ag}^{+}$in the $\mathrm{AgI}$ lattice gap from binding to electrons to form silver atoms ${ }^{21}$. The $\mathrm{E}_{\mathrm{VB}}$ of $\mathrm{BiOI}$ is higher than that of AgI. Thus, the holes in the valence band of BiOI are transferred through the interface to the valence band of AgI. The photogenerated electrons and holes are separated, and the photocatalytic efficiency is greatly improved. After light falls on the catalyst, it becomes excited and generates electron-hole pairs. The electrons $\left(\mathrm{e}^{-}\right)$interacts with the surrounding oxygen $\left(\mathrm{O}_{2}\right)$ and produces superoxide radicals $\left(\mathrm{O}_{2}^{-\bullet}\right)$. The surface of the photocatalyst contains water, which is called absorbed water. The positive holes are oxidized with water, which interacts with adsorbed hydroxyl ions $\left(\mathrm{OH}^{-}\right)$, thereby making way for the formation of the hydroxyl radicals $(\bullet \mathrm{OH})$ in the modified AgI/BiOI composites. Both of these free radicals interact with organic dye compounds and are converted to carbon dioxide $\left(\mathrm{CO}_{2}\right)$ and water $\left(\mathrm{H}_{2} \mathrm{O}\right)$. In addition, this approach allows $\mathrm{AgI}$ to remain stable under light, further improving the photocatalytic activity. Thus, the AgI/BiOI compound exhibits higher photocatalytic activity than sole AgI or BiOI. A saturation value is reached with increasing AgI content in $\mathrm{AgI} / \mathrm{BiOI}$ composites. The excess $\mathrm{AgI}$ forms a new composite center. That part of the photogenerated electrons and holes recombines, thereby reducing the catalyst activity. This finding coincides with the experimental results, i.e., the composite catalyst level first increased and then 
decreased with increasing $\mathrm{AgI}$ recombination ratio. The optimum amount of $\mathrm{AgI}$ in the $\mathrm{AgI} / \mathrm{BiOI}$ compound is $50 \%$, which shows the best photocatalytic activity in the experiment.

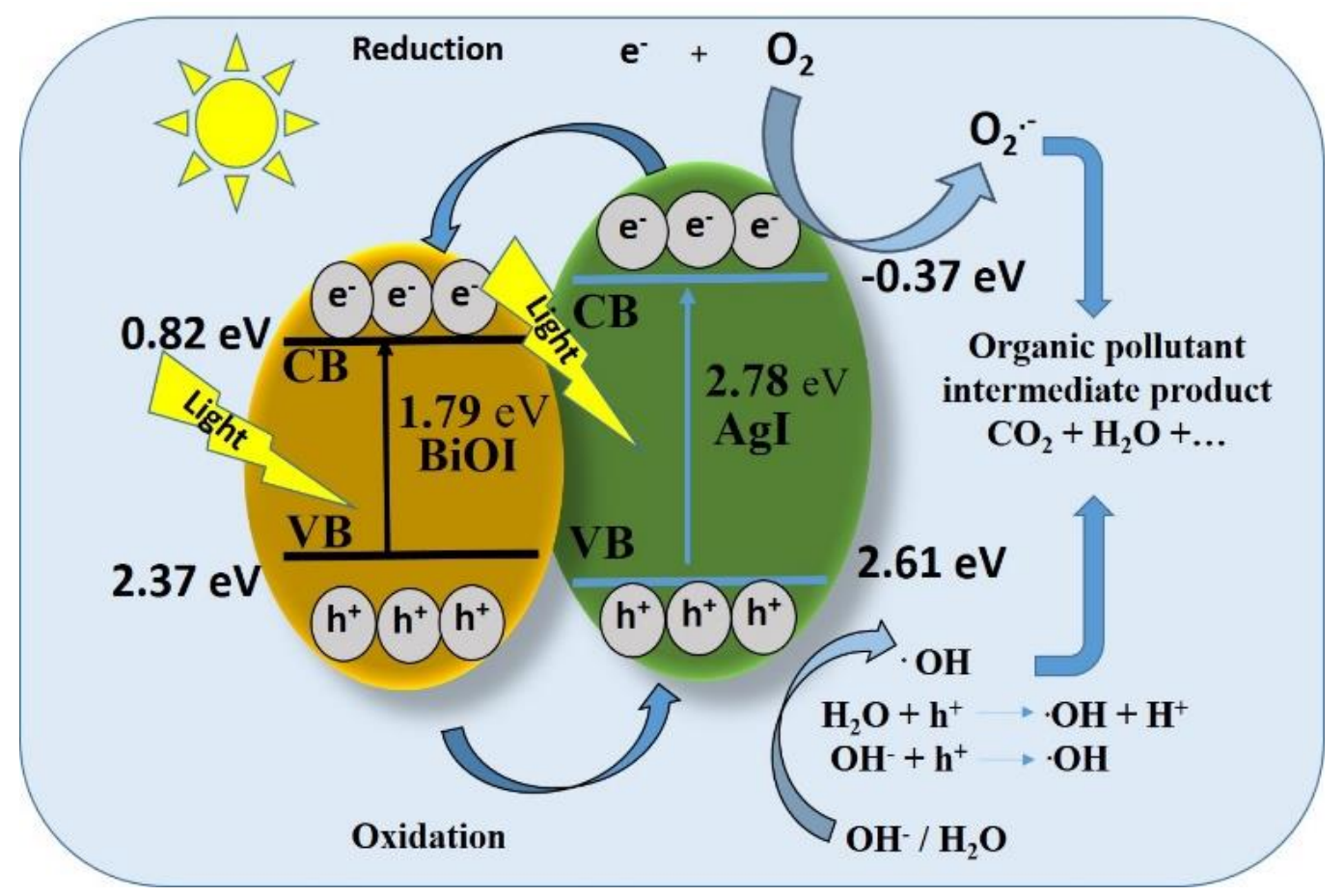

Fig. 10. Schematic illustration of the photocatalytic process of $\mathrm{AgI}$ nanoparticle/BiOl nanosheet composite.

\section{Conclusion}

Nanoparticle AgI/BiOI nanosheet composites with different composite ratios were successfully prepared by the precipitation-hydrothermal method. It had good crystallinity, high purity, and good photocatalytic performance. The $50 \% \mathrm{AgI} / \mathrm{BiOI}$ compound exhibited the best visible photocatalytic activity and degraded $96.9 \%$ and $97 \%$ of the MO and CBB dye, respectively, in 30 min, leading to near-complete degradation. The absorption edges of BiOI and AgI were $653 \mathrm{~nm}$ and $454 \mathrm{~nm}$, respectively, and they all had a visible light response. The bandgap widths ( $\left.\mathrm{E}_{\mathrm{g}}\right)$ for $\mathrm{BiOI}$ and $\mathrm{AgI}$ were 1.79 and $2.78 \mathrm{eV}$, respectively. $\mathrm{BiOI}$ and $\mathrm{AgI}$ had matching potentials, and p$\mathrm{n}$ junctions were formed at the interface. In this way, the electrons and holes were separated, and the quantum recombination rate was reduced. Moreover, the AgI was prevented from being electronically reduced to elemental silver, thereby improving the visible photocatalytic activity of 
the compound. This compound can potentially be applied to the elimination of $\mathrm{MO}$ and CBB from aqueous solutions during water treatment.

\section{Conflicts of interest}

There are no conflicts to declare.

\section{Acknowlegements}

The research reported in this publication was sponsored by the National Natural Science Foundation of China (21572180), the Intergovernmental Science and Technology Cooperation and Exchange Program between China and Romania (43-24-20180510), the Natural Science Basic Research Plan in Shaanxi Province of China (2012JZ2002), the Academic Funding of Northwestern Polytechnical University (NPU, 17GH0402, W212002). We gratefully acknowledge the Analytical and Testing Center of Northwestern Polytechnical University for providing necessary laboratory facilities to carry out the XRD, SEM, TEM, EDX and XPS analysis in this work.

\section{References}

1 S. Gao, Guo C, Hou S, Wan L, Wang Q, Lv J, Zhang Y, Gao J, Meng W, and Xu J., J.Hazard. Mater., 2017, 331, 1-12.

2 P. Wang, M. Cao, C. Wang, Y. Ao, J. Hou, and J. Qian, Appl. Surf. Sci., 2014, 290, 116-124.

3 A. B. Dos Santos, F. J. Cervantes, and J. B. Van Lier, Biores. Technol., 2007, 98(12), 2369-2385.

4 L. Gao, J. Du, and T. Ma, Ceram. Int., 2017, 43(12), 9559-9563.

5 M. Sun, Y. Wang, Y. Fang, S. Sun, and Z. Yu, J. Alloys Compd., 2016, 684, 335-341.

6 F. Zhou, H. Song, H. Wang, S. Komarneni, and C. Yan, Appl. Clay Sci., 2018, 166, 9-17.

7 M. J. Islam, H. K. Kim, D. A. Reddy,Y.Kim, R. Ma, H. Baek, J. Kim, and T. K. Kim, Dalt. Trans. 46(18), 6013-6023 (2017).

8 L. Chen, D. Jiang, T. He, Z. Wu, and M. Chen, CrystEngComm, 2013, 15(37), 7556-7563.

9 J. Hu, S. Weng, Z. Zheng, Z. Pei, M. Huang, and P. Liu, J. Hazard. Mater., 2014, 264, 293-302.

10 J. Cao, B. Xu, H. Lin, B. Luo, and S. Chen, Chem. Eng. J., 2012, 185, 91-99.

11 J. Li, Q. Zhou, F. Yang, L. J. Wu, W.Y. Li, R.P. Ren, and Y.K. Lv, New J. Chem., 2019, 43(37), 1482914840.

12 S. B. Ning, H. X. Lin, Y. C. Tong, X. Y. Zhang, Q. Y. Lin, Y. Q. Zhang, J. L. Long, X. X. Wang, Appl. Catal. B: Environ. 2017, 204, 1-10.

13 X. Xiao, R. Hao, M. Liang, X. X. Zuo, J. M. Nan, L. S. Li, W. D. Zhang, J. Hazard. Mater., 2012, 233, 122-130.

14 L. Yosefi and M. Haghighi, Appl. Catal. B: Environ., 2018, 220, 367-378.

15 C. Dong, K.-L. Wu, X.-W. Wei, J. Wang, L. Liu, and B.-B. Jiang, Appl. Catal. A: Gener., 2014, 488, 11-18. 
16 H. Cheng, B. Huang, Y. Dai, X. Qin, and X. Zhang, Langmuir, 2010, 26(9), 6618-6624.

17 J. Cao, B. Xu, B. Luo, H. Lin, and S. Chen, Appl. Surf. Sci., 2011, 257(16), 7083-7089.

18 Q. Yang, J. Huang, J. Zhong, J. Chen, J. Li, and S. Sun, Curr. Appl. Phys., 2017, 17(9), 1202-1207.

19 Q. Wang, X. D. Shi, E. Q. Liu, J. C. Crittenden, X. J. Ma, Y. Zhang, Y. Q. Cong, J. Hazard. Mater., 2016, 317, 8-16.

20 H. Cheng, H. F. Cheng, W. J. Wang, B. B. Huang, Z.Y. Wang, J. Zhan, X.Y. Qin, X. Y. Zhang, and Y. Dai, J. Mater. Chem. A, 2013, 1(24), 7131-7136.

21 Y. H. Lv, H. Liu, W. Zhang, S. L. Ran, F, L. Chi, B. Yang, A. L. Xia, J. Environ. Chem. Eng., 2013, 1(3), 526-533.

22 J. Di, J. X. Xia, M. X. Ji, B. Wang, S. Yin, H. Xu, Z. G. Chen, and H. M. Li, Langmuir, 2016, 32(8), 2075-2084.

23 X. Zhang, L. Zhang, T. Xie, and D. Wang, J. Phys. Chem. C, 2009, 113(7), 7371-7378.

24 W. Shi, H. Lv, S. Yuan, H. Huang, Y. Liu, and Z. Kang, Sep. Purif. Technol., 2017, 174, 75-83.

25 H. B. Yu, B. B. Huang, H. Wang, X. Z.Yuan, L. B. Jiang, Z. B. Wu, J. Zhang, G. M. Zeng, J. Coll. interf. sci., 2018, 522, 82-94.

26 Y. Li, J. Wang, H. Yao, L. Dang, and Z. Li, J. Mol. Catal. A: Chem., 2011, 334(1-2), 116-122.

27 S.-R. Zhu, Q. Qi, Y. Fang, W.-N. Zhao, M.-K. Wu, and L. Han, Crys. Grow. Des., 2017, 18(2), 883891.

28 J. Cao, B. Xu, H. Lin, and S. Chen, Chem. Eng. J., 2013, 228, 482-488.

29 M. J. Islam, D. A. Reddy, N. S. Han, J. Choi, J. K. Song, and T. K. Kim, Phys. Chem. Chem. Phys., 2016, 18(36), 24984-24993.

30 M. J. Islam, D. A. Reddy, R. Ma, Y. Kim, and T. K. Kim, Solid State Sci. 61, 32-39 (2016).

31 F. Guo, W. L. Shi, H. B. Wang, M. M. Han, W. S. Guan, H. Huang, Y. Liu, Z. H. Kang, J. Hazard. Mater., 2018, 349, 111-118.

32 J. Cao, B. Xu, B. Luo, H. Lin, and S. Chen, Catal. Commun., 2011, 13(1), 63-68.

33 Z. Liu, H. Ran, B. Wu, P. Feng, and Y. Zhu, Coll. Surf. A: Physicochem. Eng. Asp., 2014, 452, 109114 .

34 Y. Ao, K. Wang, P. Wang, C. Wang, and J. Hou, Dalt. Trans., 2016, 45(19), 7986-7997.

35 D. Wu, H. Wang, C. Li, J. Xia, X. Song, and W. Huang, Surf. Coat. Technol., 2014, 258, 672- 676. 


\section{Graphical abstract}
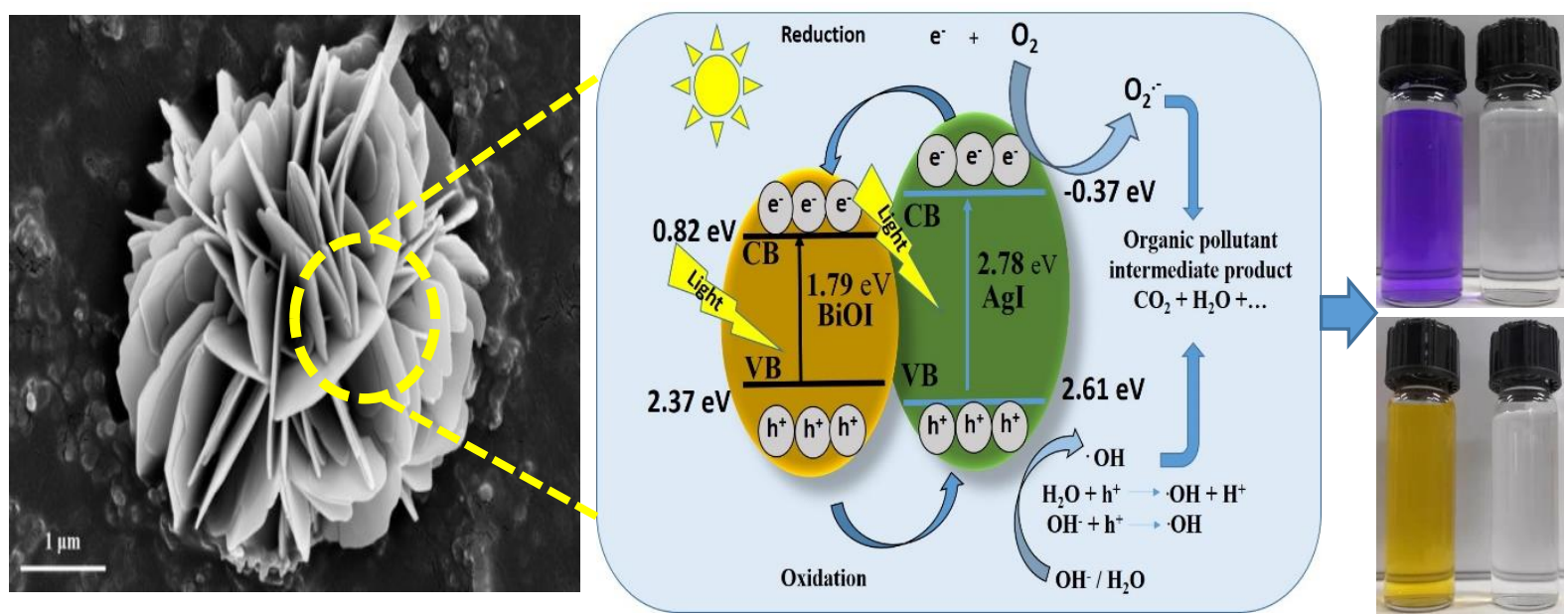

\section{Highlights}

1 Adjustment of the n-type wide bandgap of AgI with p-type narrow bandgap of BiOI.

2 A scheme was designed to prepare $\mathrm{AgI} / \mathrm{BiOI}$ composites as photocatalysts with different masses of AgI.

3 The 50\% AgI/BiOI composite showed the highest photocatalytic activity in the degradation of $\mathrm{MO}$ and $\mathrm{CBB}$ dye.

4 The $\mathrm{AgI} / \mathrm{BiOI}$ photocatalyst has a fast response to visible-light and high stability. 